\title{
Chromosomal-level genome assembly of the bioluminescent cardinalfish Siphamia tubifer, an emerging model for symbiosis research
}

\author{
Gould, AL ${ }^{1}$, JB Henderson², AW Lam² \\ 1. Ichthyology Department, Institute for Biodiversity Science and Sustainability, California \\ Academy of Sciences, 55 Music Concourse Dr. San Francisco, CA 94118 \\ *Corresponding author: agould@calacademy.org \\ 2. Center for Comparative Genomics, Institute for Biodiversity Science and Sustainability, \\ California Academy of Sciences, 55 Music Concourse Dr. San Francisco, CA 94118
}

\begin{abstract}
The bioluminescent symbiosis between the sea urchin cardinalfish Siphamia tubifer (Kurtiformes: Apogonidae) and the luminous bacterium Photobacterium mandapamensis is an emerging vertebrate-bacteria model for the study of microbial symbiosis. However, there is little genetic data available for the host fish, limiting the scope of potential research that can be carried out with this association. In this study, we present a chromosomal-level genome assembly of $\mathrm{S}$. tubifer using a combination of PacBio HiFi sequencing and $\mathrm{Hi}-\mathrm{C}$ technologies. The final genome assembly was $1.2 \mathrm{~Gb}$ distributed on 23 chromosomes and contained 32,365 protein coding genes with a BUSCO completeness score of $99 \%$. A comparison of the $S$. tubifer genome to that of another non-luminous cardinalfish revealed a high degree of synteny, whereas a similar comparison to a more distant relative in the Gobiiformes order revealed a fusion of two chromosomes in the cardinalfish genomes. An additional comparison of orthologous clusters among these three genomes revealed a set of 710 clusters that were unique to S. tubifer in which $23 \mathrm{GO}$ pathways were significantly enriched, including several relating to host-microbe interactions and one involved in visceral muscle development, which could be related to the musculature involved in the gut-associated light organ of $S$. tubifer. We also assembled the complete mitogenome of $S$. tubifer and discovered both an inversion in the WANCY tRNA gene region resulting in a WACNY gene order as well as heteroplasmy in the length of the control region for this individual. A phylogenetic analysis based on the whole mitochondrial genome indicated that $S$. tubifer is divergent from the rest of the cardinalfish family, bringing up questions of the involvement of the bioluminescent symbiosis in the initial divergence of the ancestral Siphamia species. This draft genome assembly of $S$. tubifer will enable future studies investigating the evolution of bioluminescence in fishes as well as candidate genes involved in the symbiosis and will provide novel opportunities to use this system as a vertebrate-bacteria model for symbiosis research.
\end{abstract}


Introduction

The cardinalfish genus Siphamia (Kurtiformes: Apogonidae) is comprised of 25 species, all of which are symbiotically bioluminescent. The fish has an abdominal light organ attached to the gut that harbors a dense population of a single species of luminous bacterium, Photobacterium mandapamensis, a member of the Vibrionaceae (Yoshiba \& Haneda 1967, Wada et al. 2006, Kaeding et al. 2007, Urbanczyk et al. 2011, Gould et al. 2021). Additional cardinalfish species belonging to at least three other genera are also bioluminescent, however those species produce light autogenously and do not form a symbiosis with luminous bacteria (Thacker \& Roje 2009). Members of the Siphamia genus are found throughout the Indo-Pacific, but $S$. tubifer (Figure 1) has the broadest distribution, spanning from east Africa to the French Polynesian Islands (Gon \& Allen 2012). Siphamia tubifer is also the most well-studied Siphamia species to date; previous studies have characterized the fish's life history (Gould et al. 2016), behavioral ecology (Eibl-Eibesfeldt 1961, Tamura 1982, Gould et al. 2014, 2015), and population genetics (Gould et al. 2017), as well as the the symbiosis with P. mandapamensis (Dunlap \& Nakamura 2011, Dunlap et al. 2012, Gould et al. 2019, Iwai 1958, 1971). Unlike most symbiotically luminous fish species that inhabit deep water or have pelagic life histories, $S$. tubifer is a shallow, reef-dwelling species and can be raised in aquaria, both with and without its luminous symbiont, rendering the symbiosis to be experimentally tractable (Dunlap et al. 2012). Thus, the S. tubifer-P. mandapamensis symbiosis an emerging model for the study of vertebrate-bacteria associations, and is especially well-suited for studies of the vertebrate gut microbiome.

Despite an accumulation of knowledge of the biology of $S$. tubifer and its symbiosis with $P$. mandapamensis, there is little genomic information available for the fish, limiting the scope of possible studies that can be carried out with this association. A high-quality reference genome of $S$. tubifer will unlock new research opportunities to investigate the genetic mechanisms regulating this highly specific association, further enhancing its strength as a model system. Thus, we present a chromosomal-level assembly of the genome of $S$. tubifer produced by a combination of third-generation sequencing technology (PacBio HiFi sequencing) and chromosome conformation capture methods (Hi-C, Lieberman-Aide et al. 2009, vanBerkum et al. 2010). We then compare our S. tubifer genome assembly and annotation to that of other chromosomal-level assemblies of a closely related but non-luminous cardinalfish species and a more distant relative in the sister order Gobiiformes to describe synteny between the genomes and identify candidate genes that could be involved in the symbiosis. We also present a whole mitochondrial genome assembly of $S$. tubifer and use this sequence information to infer $S$. tubifer's phylogenetic position within the cardinalfish family, providing further insight into the

\section{Methods}

80 
82

All tissue was obtained from a single female Siphamia tubifer specimen collected from a shallow reef in Okinawa, Japan $\left(26.66^{\circ} \mathrm{N}, 127.88^{\circ} \mathrm{E}\right)$. The fish was collected and euthanized following approved protocols and permits for the capture, care and handling of fish by the California Academy of Science's Institutional Animal Care and Use Committee. Immediately following euthanasia, fresh muscle tissue was sampled from the flanking region of the fish for high molecular weight (HMW) DNA extraction using a phenol-chloroform extraction protocol provided by Pacific Biosciences of California, Inc. Fresh muscle and brain tissue were also sampled from the same individual for $\mathrm{Hi}-\mathrm{C}$ methods. The HMW DNA was prepared for PacBio HiFi sequencing at UC Berkeley's QB3 Genomics Sequencing Lab (Berkeley, CA) and sequenced on one Sequel II 8M SMRT Cell.

\section{Hi-C library preparation and sequencing}

In situ $\mathrm{Hi}-\mathrm{C}$ libraries were prepared from the freshly homogenized muscle and brain tissues following the protocol described in Rao et al. (2014) with slight modifications. After the Streptavidin pull-down step, the biotinylated $\mathrm{Hi}-\mathrm{C}$ products underwent end repair, ligation, and

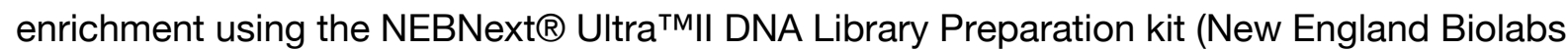
Inc, Ipswich, MA). Titration of the number of PCR cycles was performed as described in Belton et al. (2012). The final libraries were then sequenced as paired-end 150 bp reads on the Illumina NovaSeq 6000 platform by Novogene Corporation, Inc. (Sacramento, CA).

\section{Genome size estimation, assembly and chromosome mapping}

Circular Consensus Sequences (CCS) were generated using ccs v5.0.0 (https://github.com/PacificBiosciences/pbbioconda), from 35.95M subreads, representing 442.25G bases, and filtered to produce HiFi reads, defined as having at least two circular passes and minimum of $99.9 \%$ accuracy. A custom script created a fastq file containing the HiFi reads extracted from the .bam output file of the ccs step. Jellyfish (Marcais \& Kingsford 2012) was then used to count and create histograms of kmers size 21 and 25 from the HiFi reads, and GenomeScope v2.0 (Ranallo-Benavidez et al. 2020) was run on each set to determine estimates of genome size.

Next, filtering was performed to remove contaminant sequences. Since using blastn (Altschul et al. 1990) and other similar tools is inefficient with long reads, we first used minimap2 (Li 2018) with the genome of the closely related orbiculate cardinalfish, Sphaeramia orbicularis, to exclude matching reads from further contaminant analysis. For the remaining sequences, blastn was then used against a database of Siphamia tubifer's luminous symbiont, Photobacterium mandapamensis (Urbanczyk et al. 2011), to identify its sequences as contaminants. Additionally, to further reduce the analysis, the first 500 bases of the remaining long reads were used as blastn queries against the nt database with option -taxidlist restricting search to bacteria, and those excluded with e-value greater than -1e10. Similarly, mitochondrial DNA sequences were identified and removed for separate analysis by using 
blastn against a database of three Apogonidae mitochondrial genomes: S. orbicularis, Ostorhinchus fleurieu, and Pristicon trimaculatus. Subsequent nuclear genome analysis used the remaining long read HiFi sequences with contaminant and mitochondrial sequences removed.

The remaining HiFi sequences were assembled with hifiasm v0.13-r308 (Cheng et al. 2021). The hifiasm assembly program is designed for HiFi reads produced from a diploid genome and also incorporates purge_dups (Guan et al. 2020) to separate out duplicate haplotigs, producing a primary assembly of the higher quality contigs and an alternate assembly of contigs including the duplicates. For comparison, we also ran Improved Phase Assembler, ipa v1.3.0, (PacificBiosciences 2020) to create an assembly from the same input. We then ran quickmerge v0.3 (Chakraborty et al. 2016) for a third assembly where the hifiasm result was used as the query and the ipa output as a reference assembly to attempt to bridge gaps in the hifiasm genome representation.

The $\mathrm{Hi}-\mathrm{C}$ reads, consisting of $624.35 \mathrm{M}$ combined brain and muscle tissue read pairs, were mapped using juicer v1.6 (Durand et al. 2016b) against the hifiasm assembled contig level genome. We next ran 3d-dna v180922 (Dudchenko et al. 2017) with its early-exit flag to create an input file for JuiceBox Assembly Tools (JBAT) (Durand et al. 2016a, Dudchenko et al. 2018) that represents the assembly with contigs ordered and oriented in a candidate chromosomal level depiction. Using JBAT, we interactively updated location and orientation of contigs and their delineation at the chromosome level (Figure 2a). This assembly was also queried against the nt database using blastn to identify any additional contaminants for removal.

To assess the level of genome completeness, we ran BUSCO v5.12 (Simão et al. 2015) with the 3,640 entry Actinopterygii dataset in both its MetaEuk (Karin et al. 2020) and AUGUSTUS (Keller et al. 2011) modes. We then used a custom script to update BUSCOs found by AUGUSTUS that were missing in the MetaEuk results and another to report the combined BUSCO scores.

\section{Gene annotation and synteny}

Prior to gene annotation, de novo repeats were identified from the $S$. tubifer genome assembly using RepeatModeler v2.0.1(Flynn et al. 2020). First, the fasta file representing these species specific repeat models and the vertebrate repeat models from Repbase (https://www.repeatmasker.org) RepeatMasker libraries v20181026 were appended into a combined file. This file was then used as the input library to Repeatmasker v4.0.9 (Smit et al. 2013-2015) with the options -small -xsmall and -nolow to create the soft-masked repeat version of the assembly file used for gene model annotation. BRAKER2 (Brüna et al. 2021), using GeneMark-EP+ (Brůna et al. 2020) and AUGUSTUS, combined with the vertebrate protein database from OrthoDB v10 (https://www.orthodb.org) (Kriventseva et al. 2019), was 
used for gene annotation. The output of potential gene models represented in .gff3, amino acid, and DNA files were subject to additional filtering together with functional annotation.

To check for protein domains, we ran InterProScan v5.51-85.0 (Jones et al. 2014) on the amino acid sequences found in the BRAKER2 results. These sequences were also used as queries for a blastp run on three databases: SwissProt, TrEMBL, and the vertebrate proteins from OrthoDB v10. The DNA versions of the sequences were also queried with blastn against the nt database downloaded on February 13, 2021. Gene models, in .gff3, amino acid, and DNA files, were kept for those sequences with an InterProScan determined protein domain and one of the four database searches yielding a match with an e-value $0.1 \mathrm{e}-6$ or less. These files were then updated with the matching descriptions indicating they were similar to the highest scoring match of the four searches. Protein domain IDs and Gene Ontology (GO) terms, as determined by the InterProScan output, were added to the .gff3 file for each retained gene model as was the functional annotation description. tRNAscan-SE v2.0.8 (Chan et al. 2021) was implemented to identify tRNAs throughout the genome.

We then compared the genome annotation of $S$. tubifer to that of the closely related nonluminous cardinalfish, Sphaeramia orbicularis, and to a more distant member of the sister order Gobiiformes, the mudskipper Periophthalmus magnuspinnatus, using OrthoVenn2 (Xu et al. 2019). We determined the number of shared and unique protein clusters among these species and carried out a GO enrichment analysis on the unique clusters identified for S. tubifer. Next, we examined synteny between our S. tubifer genome assembly and the chromosomal-level genomes of both S. orbicularis (GenBank GCF_902148855.1) and P. magnuspinnatus (GenBank GCA_009829125.1) using the set of single copy orthologs identified from the BUSCO (Simão et al. 2015) Actinopterygii gene set and converted the output for visualization in Circos (Krzywinski et al. 2009) using custom scripts.

\section{Mitochondrial genome assembly and analysis}

Mitochondrial genome analysis was based on sequences matching at least $60 \%$ query coverage in a blastn match (qcovus format specifier) to one of the three Apogonidae mitochondrial genomes; S. orbicularis, O. fleurieu, and $P$. trimaculatus. When matched to the reverse strand, sequences were reverse complimented and_RC was appended to the name, resulting in all sequences having the same strand orientation. Megahit (Li et al. 2015) was then run on these sequences to assemble a draft mitogenome and MITOS2 (Bernt et al. 2013) was used to annotate the mitogenome.

GenBank annotations for the three Apogonidae mitogenomes were downloaded and their sequences were extracted into .fasta files containing records corresponding to the genome's rRNAs, tRNAs, and protein coding genes. The $S$. tubifer mitochondrial HiFi reads were queried with a subject database of these sequences from the three mitogenomes using blastn with its task blastn option (overriding default -task megablast). These matches were then used to split 
reads into three sets of new .fasta records using tRNA Phe and Pro as markers: (a) Phe to Pro (or end of read if no Pro), (b) if no Phe, then beginning of read to Pro, (c) Pro to Phe when both found, capturing the complete control region in between. The first 2 sets were used for tRNA analysis, including tRNA order, and the third set was used for control region repeat and heteroplasmy analysis. Mitfi (Jühling et al. 2012) was used to identify tRNAs from 176 reads from sets (a) and (b) that matched at least $90 \%$ query coverage to one of the three closely related species' mitogenomes. Tandem Repeat Finder (TRF) (Bensen 1999) was run to find repeats in the control region set.

Using the whole mitochondrial genome assembly, the phylogenetic placement of $S$. tubifer within the cardinalfish family was inferred. This analysis also included one Kurtus species and several species of gobies for reference, as well as two members of the Syngnathiformes order as an outgroup. Whole mitochondrial sequences (excluding the control regions) were aligned using MAFFT (Katoh et al. 2002), and the aligned reads were used to construct maximum likelihood trees with raxml-ng (Kozlov et al. 2019) using the substitution model with the lowest BIC score as predicted by IQtree (Nguyen et al 2015) and 500 bootstrap replicates.

\section{Results}

\section{Genome size estimation, assembly, and chromosome mapping}

A total of 2,110,443 HiFi CCS reads consisting of 27,799,385,228 bp were generated from the single HiFi library, with a polymerase N50 of 183,061 and subread N50 of 13,439 . Over $97 \%$ of the $\mathrm{HiFi}$ reads were between 12,000 and 15,000 bp. From these sequences, the GenomeScope size estimate, using kmer lengths 21 and 25, ranged from 947,587,691 to $964,260,239 \mathrm{bp}$. Repeat length was estimated as $215,783,447$ to $256,391,534 \mathrm{bp}$, though repeat length is often underestimated by kmer counting models, leading to a lower overall estimate of genome size. After contaminant and mitochondrial sequence removal 2,109,973 sequence reads were left with 6,158,291 bp excluded from the source HiFi reads. These remaining sequences were used as input for the assembly programs hifiasm and ipa. Based on contiguity and accuracy metrics, we used the hifiasm assembly to scaffold with the Hi-C reads.

For the Hi-C libraries, a total of $742,280,226$ and $506,411,380$ reads were produced from the muscle and brain tissue, respectively. Of those, $100 \%$ of the muscle reads and $99.98 \%$ of the brain reads were clean and of high quality, resulting in GC contents of $39.3 \%$ and $43.9 \%$. The Juicer mapping program found $245,145,667$ read pairs having $\mathrm{Hi}-\mathrm{C}$ contacts. After interactive modification with JBAT, guided by the $3 d$-dna program contig placement and orientation, the resulting genome assembly was $1.2 \mathrm{~Gb}$ distributed on 23 chromosomes, and $1.81 \%$ unplaced scaffolds, with a contig N50 of 2.3 Mb and scaffold N50 of 51.1 Mb (Table 1), and 37.71\% GC content. There are 1,960 contigs constituting chromosomal sequences. An additional two dozen smaller contig records were identified as contaminants by the final nt blastn search (primarily Arthropoda, though of unknown origin) and were removed to produce the final 
assembly. This assembly has the same summary statistics reported above except for the unplaced scaffold percentage, which was slightly lower (1.74\%).

The 23 chromosomes in the S. tubifer genome assembly are numbered 1 to 22 and 24 based on synteny with another cardinalfish genome, the 23 chromosome S. orbicularis genome assembly fSphaOr1.1 (GenBank GCF_902148855.1), which is based on synteny with the 24 chromosome medaka genome (GenBank ASM223467v1), representing the fusion of the medaka chr23 into a cardinalfish chromosome.

BUSCO completeness assessment from the 3,640 entry Actinopterygii dataset show 99\% complete with just 13 of the genes not found (MetaEuk mode: 98\% complete, AUGUSTUS mode: $97.2 \%$ complete).

\section{Genome annotation and statistics}

Repeat analysis indicated $626,216,533 \mathrm{bp}$, or $52.11 \%$ of the genome, classified as repeats, of which, most (23.7\% of the genome) are DNA repeat elements. Additionally, $7.03 \%$ of the genome contains long interspersed nuclear elements (LINEs), with $16.28 \%$ of the genome characterized as unclassified repeats. The extent of repeats may account for the discrepancy between the assembly size and the GenomeScope estimates using kmer counts.

Gene annotation identified 30,117 gene models with a total length of 360,171,123 bp, (29.99\% of the genome). Exons at 53,076,342 bp are 4.42\% of the genome and average 9.64 per gene; fewer than $10 \%$ are single exon genes. Additional per chromosome details of genes, exons, and introns are outlined in Table 2. The orbiculate cardinalfish, S. orbicularis (GenBank Annotation Release 100 2019-08-03), was the closest functional annotation reference for $17,079(56.7 \%)$ of the 30,117 S. tubifer gene models. This was followed by several other fish species: Lates calcarifer $(n=2,317)$, Seriola dumerili $(n=1,357)$, Larimichthys crocea $(n=995)$, and Stegastes partitus $(\mathrm{n}=779)$.

The orthologous cluster analysis indicated that a much lower number of protein clusters were shared between $S$. tubifer and the mudskipper $P$. magnuspinnatus $(n=419)$ than with the other cardinalfish S. orbicularis $(n=1,743)$. However, $S$. orbicularis shared a much larger number of clusters with $P$. magnuspinnatus $(\mathrm{n}=1,484)$ than did $S$. tubifer. There were also 710 unique protein clusters that were present only in the $S$. tubifer genome (Figure 3a), of which 506 were assigned to GO categories (Table S1). Overall, the largest percent of these clusters were categorized as biological processes (GO:0008150) (26\%) and cellular processes (GO:0009987) (16\%), and another $8 \%$ and $5 \%$ were identified as response to stimulus (GO:0050896) and developmental processes (GO:0032502), respectively (Figure 3b). The largest cluster was made up of 70 proteins assigned as DNA integration (GO:0015074), and the second largest cluster contained 41 proteins relating to visual perception (GO:0007601). There were also 9 genes 
significantly enriched ( $p>0.01$ ). Of those, several were related to viral penetration (G0:0075732) and integration (GO:0044826) into a host as well as visceral muscle development (GO:0007522) (Table 3).

Genome synteny

Overall a high degree of synteny between the genomes of $S$. tubifer and the nonluminous, orbiculate cardinalfish S. orbicularis was observed (Figure 4a). Of the 3,555 orthologous genes from the BUSCO set only $2.5 \%(n=90)$ changed chromosomal assignment. A comparison to a more distantly related fish species, the mudskipper $P$. magnuspinnatus, a member of the sister order Gobiiformes revealed that a merge occurred between $P$. magnuspinnatus chromosomes 12 and 23 to become chromosome 12 in both cardinalfish genomes (Figure 4b). Thus, the mudskipper genome has one more chromosome $(n=24)$ than both $S$. tubifer and S. orbicularis $307 \quad(n=23)$.

\section{Mitochondrial genome}

There were 5,124,329 total bp in the $392 \mathrm{HiFi}$ reads that matched the cut-off of $60 \%$ query coverage used in the mitochondrial sequence analysis. Assuming a mitogenome is between 16,000 and $18,000 \mathrm{bp}$, this represents $285-320 x$ coverage. There were 176 reads in which $90 \%$ or greater of the read length was covered containing 2,302,235 bp.

The complete mitochondrial genome averaged $17,905 \mathrm{bp}$, but varied due to heteroplasmy in the length of the control region (Figure 5a). There were 13 protein coding genes, 22 tRNA genes, and 2 rRNA genes, as expected for a vertebrate mitogenome. However, there was an inversion of two genes detected within the region that codes for five mitochondrial tRNAs (tryptophan, alanine, asparagine, cysteine, and tyrosine), known as the WANCY region, resulting in the order of these genes to appear as WACNY (Figure 5a). Their order was determined by Mitfi annotation of the $176 \mathrm{HiFi}$ reads. All of the reads had enough tRNAs to affirm the WACNY order; 174 encompassed all of these 5 tRNA genes, and the other two reads began with CNY and NY, also indicating the WACNY gene order. There were also $135 \mathrm{HiFi}$ read excerpts that encompassed the Pro tRNA gene, the entire control region (CR), and the Phe tRNA gene from which we determined the CR lengths (excluding the Pro and Phe sequences). The length of the CR ranged from 2,620 to 6,544 bp with a mean of 4,243 bp (median $=4,317 \mathrm{bp}$ ) (Figure $5 \mathrm{~b}$ ). Of the 135 sequences, 130 had a 60 bp repeat beginning after the Pro tRNA (consensus sequence: CCCCCCGTTCGGGCTITGCTTAAGTCCATGCTAATATATTCCTIIIITCGTCCGCA), and the other 5 reads had similar repeats. This sequence, or a 1 to 4 nucleotide indel or SNP variation of it, was repeated just under twice up to 69 times in each read. A goose hairpin sequence (Quinn \& Wilson 1993), in this case $\mathrm{C}_{7} \mathrm{TAC}_{7}$, was found in 133 of the $135 \mathrm{CR}$ sequences (the two others had $\mathrm{C}_{7} \mathrm{TCAC}_{7}$ and $\mathrm{C}_{7} \mathrm{TAC}_{4} \mathrm{CAC}_{8}$ ). All of the hairpins started between 350-360 bp from the end of the CR region (the base before the start of tRNA Phe), with 105 of them 353 bp or 354 bp from the end (Figure 5a). 
The maximum likelihood phylogeny based on whole mitochondrial sequences (excluding the control region) indicates that Siphamia tubifer is divergent from rest of the Apogonidae but a member of the Apogonoidei clade, which also contains the Kurtus genus and is sister to the Gobioidei clade (Ghezelayagh et al. 2021) (Figure 6). The placement of S. tubifer as divergent from the other apogonids is also observed when analyzing a concatenation of several mitochondrial genes, excluding the WANCY tRNA genes (Figure S1). An analysis of COI on its own, however, does not align with the other tree topologies, nesting Siphamia tubifer within the cardinalfishes, sister to Ostorhinchus novemfasciatus, although with low bootstrap support (Figure S1).

\section{Discussion}

Combining PacBio HiFi sequencing with $\mathrm{Hi}-\mathrm{C}$ technology, we assembled a high-quality, chromosome-level genome for the symbiotically luminous cardinalfish Siphamia tubifer. The BUSCO score of $99 \%$ completeness indicates that this is a near complete genome and will thus serve as a valuable resource for future studies, particularly as the bioluminescent symbiosis between $S$. tubifer and $P$. mandapamensis continues to develop as a tractable, binary model system for symbiosis research. This is only the second cardinalfish genome assembly to date, and our comparison of the two indicates there is significant synteny between them, despite the divergence of $S$. tubifer from the rest of the family. An additional comparison to a more distant genome belonging to the sister order Gobiiformes, revealed a merging of two chromosomes resulting in one fewer chromosome in the cardinalfish genomes. This chromosome fusion also supports the lack of a chromosome 23 in the labelling of the $S$. orbicularis chromosomes, which were named based on synteny with the medaka genome. Determining whether this is a common feature of all cardinalfish genomes and when this merge occurred would require additional chromosomal-level genome assemblies for species in the two orders.

The orthologous cluster analysis between S. tubifer, a non-luminous cardinalfish species, and a more distant relative in the Gobiiformes order revealed 710 protein clusters unique to $S$. tubifer. Among these unique clusters, there could be candidate genes that play an important role in the bioluminescent symbiosis. In particular, several clusters were assigned GO terms with functions relating to the immune system and interactions between organisms. There were also several GO terms relating to virus-host interactions that were significantly enriched in the unique protein clusters identified for $S$. tubifer. Although it would require further investigation, the genes involved could play a role in the fish's interaction with the luminous symbiont. Also of note, visceral muscle development was enriched in the set of unique $S$. tubifer genes. The disc-shaped light organ of $S$. tubifer develops as an outcropping of the gut epithelia and is covered by a lens composed of bundles of transparent muscle tissue on its ventral side.

377 Translucent musculature known as the diffuser also runs along the ventral surface of the fish

378 from the caudal peduncle to the throat, which acts to disperse the light produced by the 
bacteria inside the light organ (Iwai 1971, Dunlap \& Nakamura 2011). The genes associated with the visceral muscle development clusters enriched in S. tubifer could be responsible for the development of the light organ and its associated musculature. Additionally, the second largest protein cluster unique to $S$. tubifer was associated with visual perception. There is large overlap in the genes expressed in the light organ and eyes of the symbiotically luminous squid, Euprymna scolopes, and the genetic signature specific to the squid light organ includes crystallin and reflectin genes, both of which are typical features of the vertebrate eye (Belcaid et al. 2019). Thus, the large cluster of proteins relating to visual perception unique to $S$. tubifer could similarly be related to genes associated with the light organ, such as crystallin and reflection genes. Future studies are needed to determine if the same overlap between the light organ and eye transcripts exist for S. tubifer.

A byproduct of $\mathrm{HiFi}$ reads for vertebrates, and many bilaterians, is the large percentage of mitogenome sequence captured in an individual read (Formenti et al. 2021). These genomes are typically in the range $\sim 16,000$ to $\sim 22,000 \mathrm{bp}$, and their GENBANK annotations canonically start at tRNA Phe and end at the control region, which makes them amenable to discover reordering, duplicated regions leading to pseudo-genes, duplicated control regions, control region repeats, and heteroplasmy associated with those and other elements of the mitogenome. With 176 mitochondrial HiFi reads in this study, each a significant percentage of the mitogenome, we were able to determine the unique WACNY ordering of the vertebrate WANCY region of tRNAs of this individual not reported in 3,034 MitoFish website annotations (downloaded June 3, 2021 from http://mitofish.aori.u-tokyo.ac.jp). However, mitochondrial gene-order rearrangements have been observed multiple times in teleost fishes (e.g. Inoue et al 2003, Poulsen et al. 2013), including rearrangements in the WANCY region. For example, a WNCAY tRNA gene order was observed for the blunt snout smooth-head Xenodermichthys copei and was most parsimoniously explained by duplications of parts of the $\mathrm{mt}$ genome with subsequent deletions (Poulsen et al. 2019). Additional sequencing of the mitochondrial genomes of more S. tubifer specimens as well as other Siphamia species would indicate whether the WACNY gene order observed in this study is unique to this individual or is a common feature of this species or genus.

PacBio HiFi reads have lower error rates than earlier long read technology, though of course errors exist and homopolymer miscalls are a known class of these. It is likely that some of the differences in the 135 reads that incorporated an entire control region, flanked by the expected tRNAs, come from sequencing error and not the control region itself. However, given that the final part of the $C R$, which is not repetitive, varies much less in length and sequence than the repeat section of the $C R$, and the fact that there are almost 4,000 bp between the smallest and largest representations (and over 2200 bp between second smallest and second largest), repeat expansion and/or contraction is likely occurring in the mitochondria of this organism. Heteroplasmy in the length of the control region has been documented for other fish species, including the three-spined stickleback (Stärner et al. 2004), two species of sardines (Samonte et al. 2000), the flatfish Platichthys flesus (Hoarau et al. 2002), and several sturgeon species 
region could be a more common occurrence that has been overlooked with previous sequencing approaches. Thus, the ability of HiFi reads to reveal heteroplasmy in the mitogenome could lead to increased observations of this phenomenon in other organisms (e.g. Formenti et al. 2021) as HiFi sequencing becomes more widely implemented. Importantly, variability in the length of the control region has previously been used as a genetic marker to discriminate between species (e.g. Faber \& Stepien 1998, Turanov et al. 2019). If heteroplasmy in the control region is a more common occurrence, then its use as a marker could be erroneous in many cases.

The phylogenetic analysis based on whole mitochondrial genome sequences indicated that $S$. tubifer is divergent from the other members of the cardinalfish family Apogonidae, a placement previously supported and estimated to have occurred approximately 50 million years ago (Thacker 2014). The evolutionary relationship of S. tubifer as sister to the rest of the cardinalfishes raises the possibility that the bioluminescent symbiosis with $P$. mandapamensis played a role in the host's initial divergence and speciation from a common ancestor. The acquisition of bacterial endosymbionts was proposed nearly a century ago as a primary mechanism by which new species can arise (Wallin 1927), and speciation by symbiosis has since been documented, primarily for several insect hosts (Brucker \& Bordenstein 2012). for Siphamia. The high-quality genome assembly for S. tubifer presented here will serve as a valuable resource for both the study of the evolution of symbiotic bioluminescence in fishes as well as the functional genomics of the symbiosis, further establishing the $S$. tubifer- $P$. mandapamensis association as a tractable model for the study of vertebrate-bacteria interactions and microbial symbiosis more broadly.

\section{Data Accessibility}

Genome assembly and associated sequencing data are available under NCBI Bioproject PRJNA736963.

\section{Author Contributions}

460 the submitted version of this manuscript.

ALG conceived of the project and secured funding for the work. ALG carried out tissue dissections and AWL preformed the high molecular weight DNA extractions and HI-C library preparations. JBH carried out the genome assembly and associated bioinformatics. ALG performed the phylogenetic analyses and data analyses. ALG and JBH contributed to the discussion and interpretation of the results and writing of the manuscript. All authors approve of

\section{Funding}


464 Funding was provided by the National Institutes for Health (NIH-DP5-OD026405-01).

465

466 Conflict of Interest

467

468 The authors declare that the research was conducted in the absence of any commercial or 469 financial relationships that could be construed as a potential conflict of interest. 
bioRxiv preprint doi: https://doi.org/10.1101/2021.09.03.458932; this version posted September 4, 2021. The copyright holder for this preprint (which was not certified by peer review) is the author/funder, who has granted bioRxiv a license to display the preprint in perpetuity. It is made available under aCC-BY-NC-ND 4.0 International license.

\section{Figures and Tables}

471

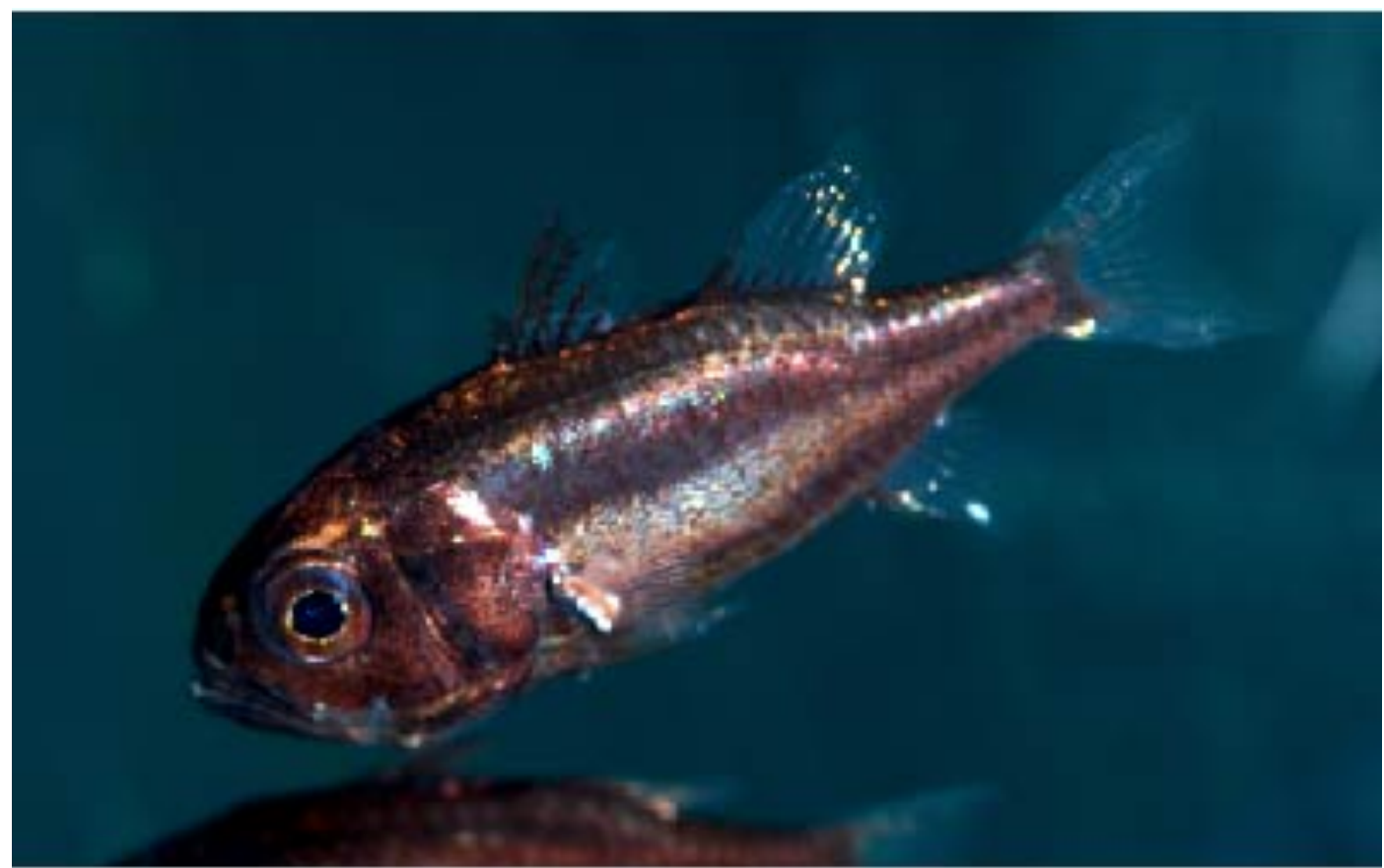

Figure 1. Photograph of Siphamia tubifer. Credit: Tim Wong, Steinhart Aquarium, California 474 Academy of Sciences.

475 
bioRxiv preprint dol: https://doi.org/10.1101/2021.09.03.458932; this version posted September 4, 2021. The copyright holder for this preprint (which was not certified by peer review) is the author/funder, who has granted bioRxiv a license to display the preprint in perpetuity. It is made available under aCC-BY-NC-ND 4.0 International license.

a.

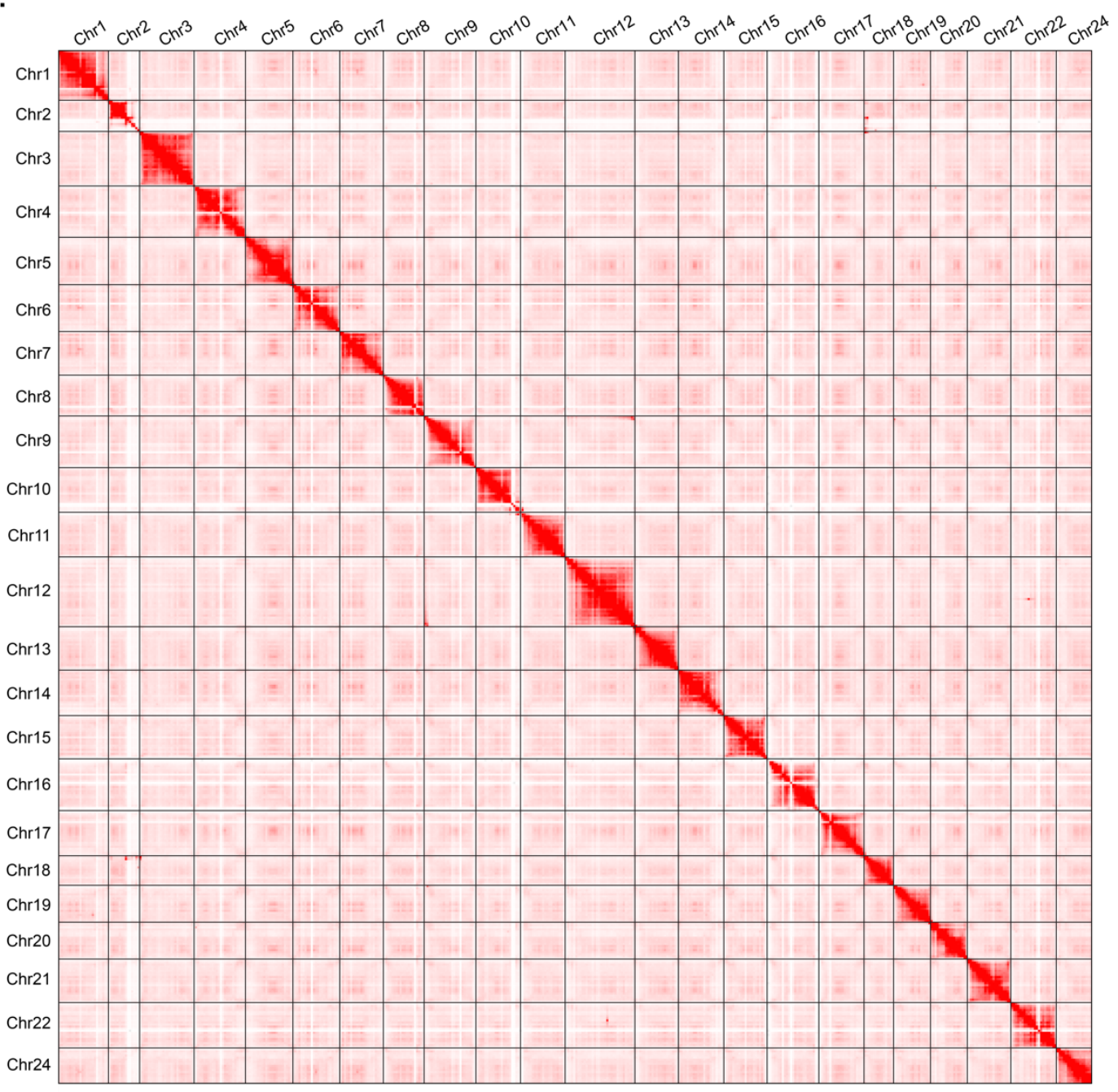

b.

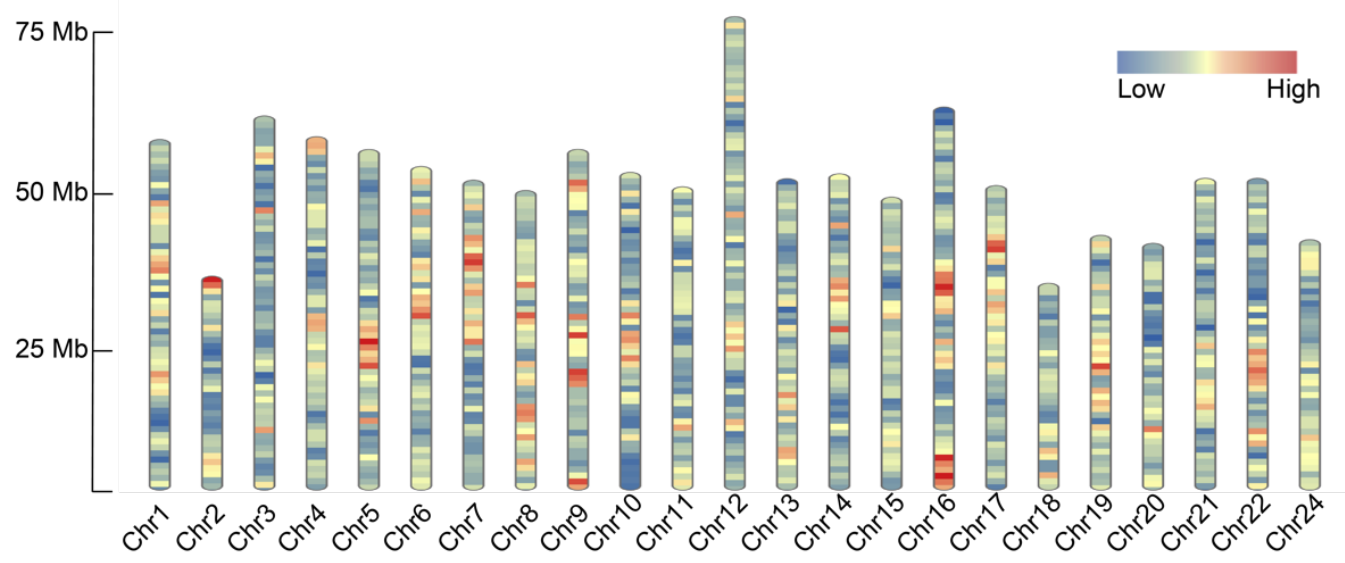

476

Figure 2. a) Hi-C contact heatmap for Siphamia tubifer. Black lines indicate chromosome

478 boundaries. b) Gene density distributed across the 23 chromosomes of the $S$. tubifer genome.

14 
a.

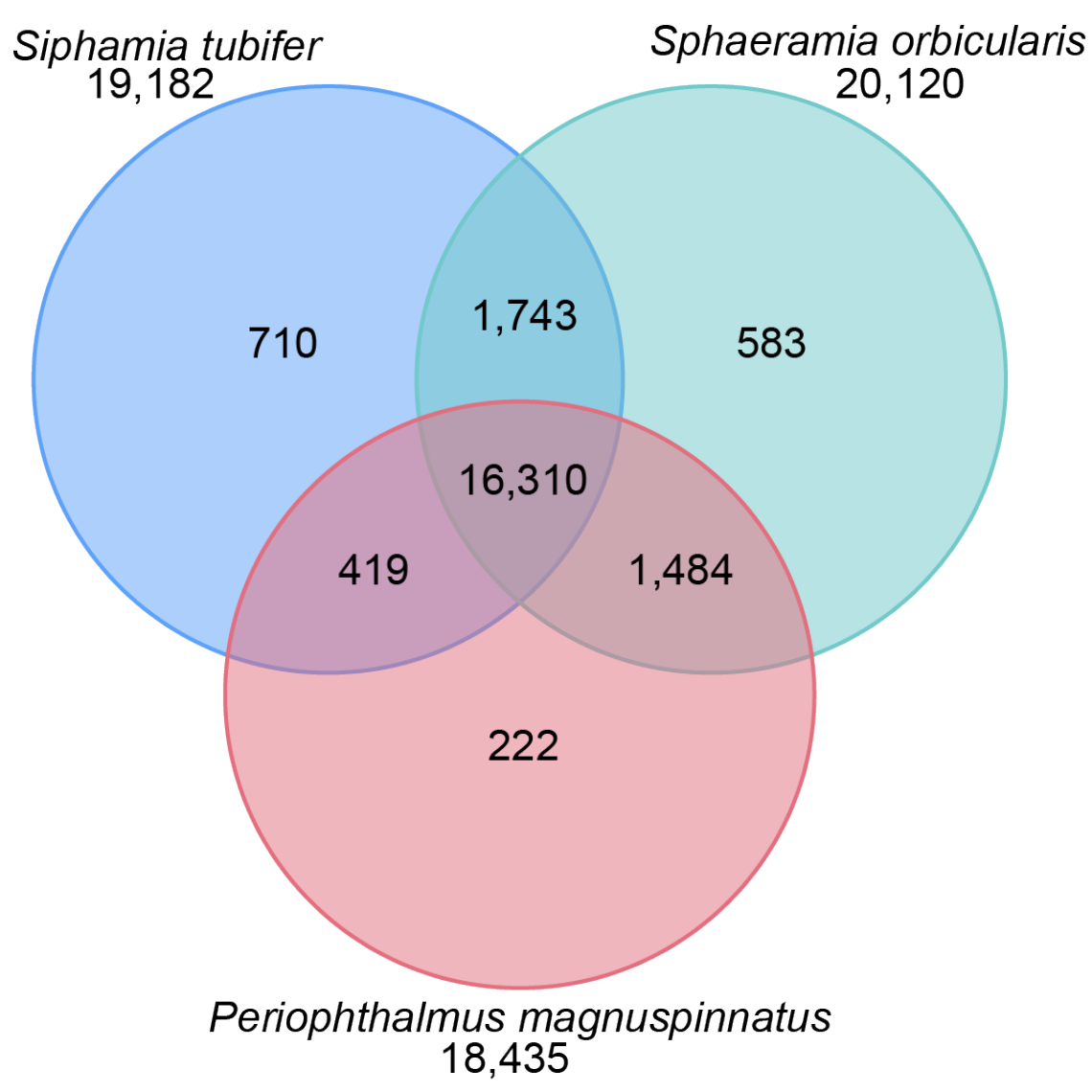

b.

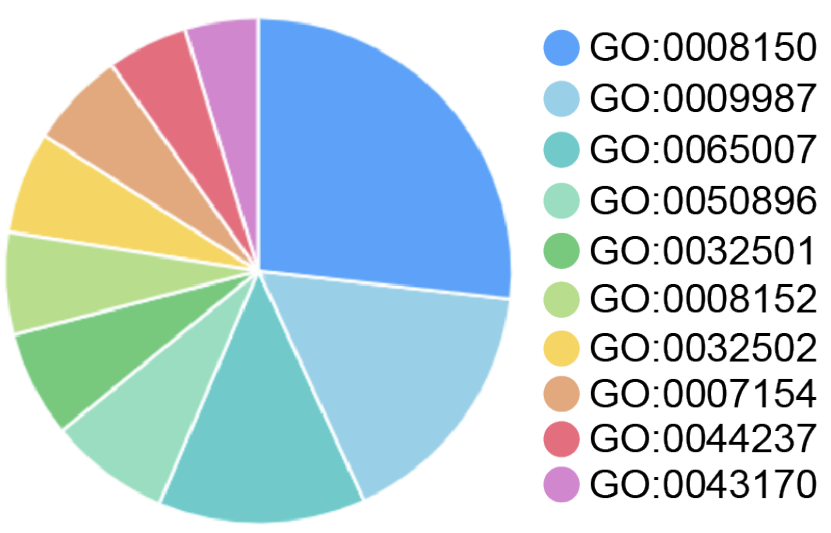

c.

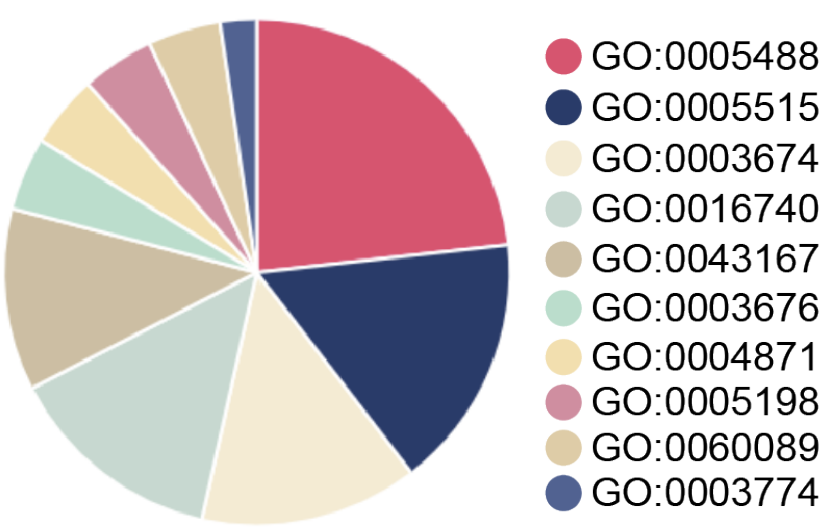

Figure 3. a) Venn diagram of the distribution of orthologous clusters among Siphamia tubifer, the non-luminous cardinalfish Sphaeramia orbicularis, and the mudskipper Periophthalmus magnuspinnatus (order Gobiiformes). b) Distribution of the top ten biological process GO terms assigned to the 710 unique clusters identified for S. tubifer and c) the top ten molecular function GO terms assigned to the gene clusters 

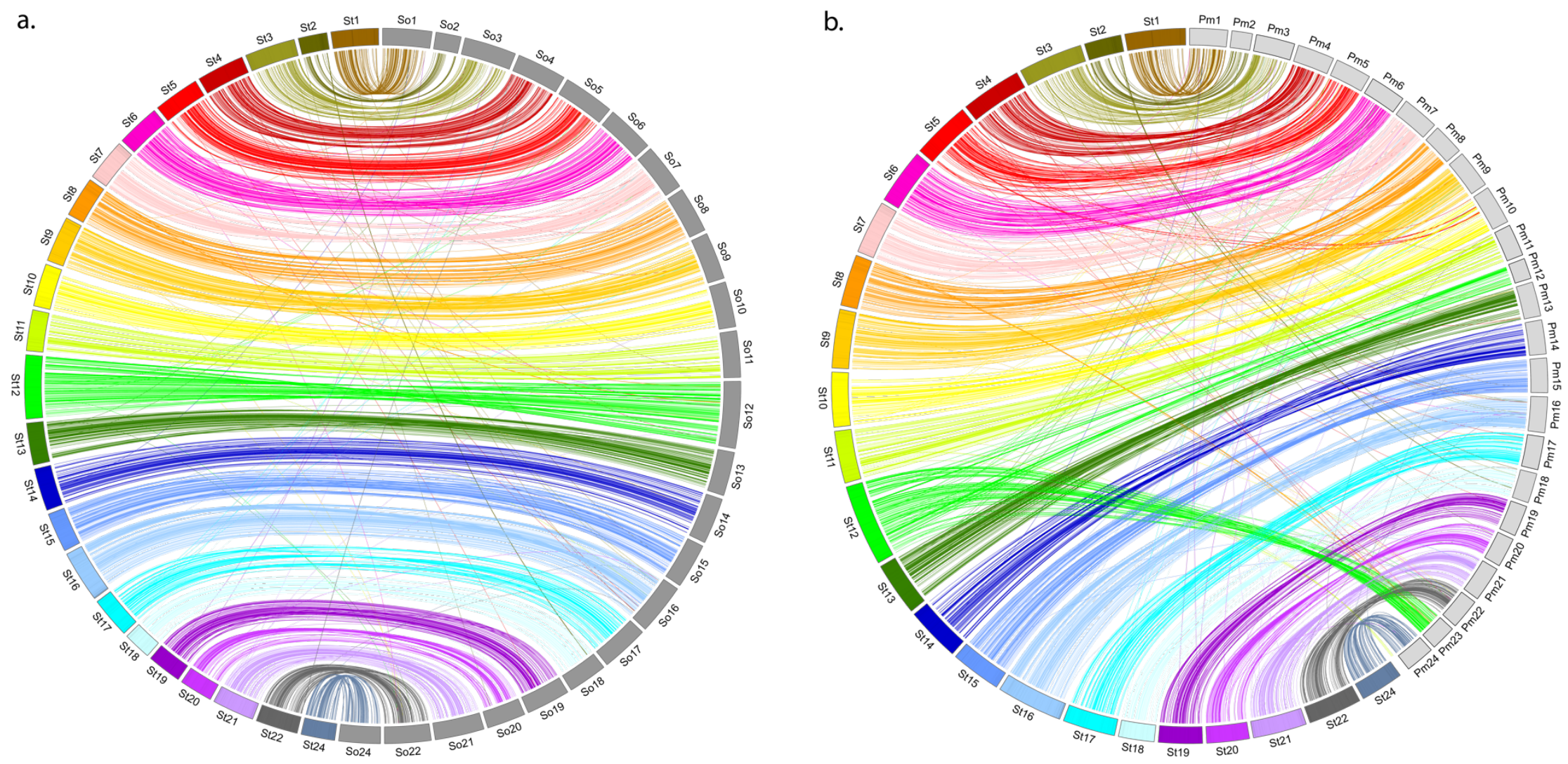

Figure 4. Circos plots depicting synteny between the genomes of Siphamia tubifer and a) the orbiculate cardinalfish, Sphaeramia orbicularis and b) the mudskipper Periophthalmus magnuspinnatus. Each chromosome in the S. tubifer genome is represented by a distinct color whereas the S. orbicularis and $P$. magnuspinnatus chromosomes are shown in dark and light gray, respectively. Links between single copy orthologs from the BUSCO Actinopterygii gene set are shown. 
a.

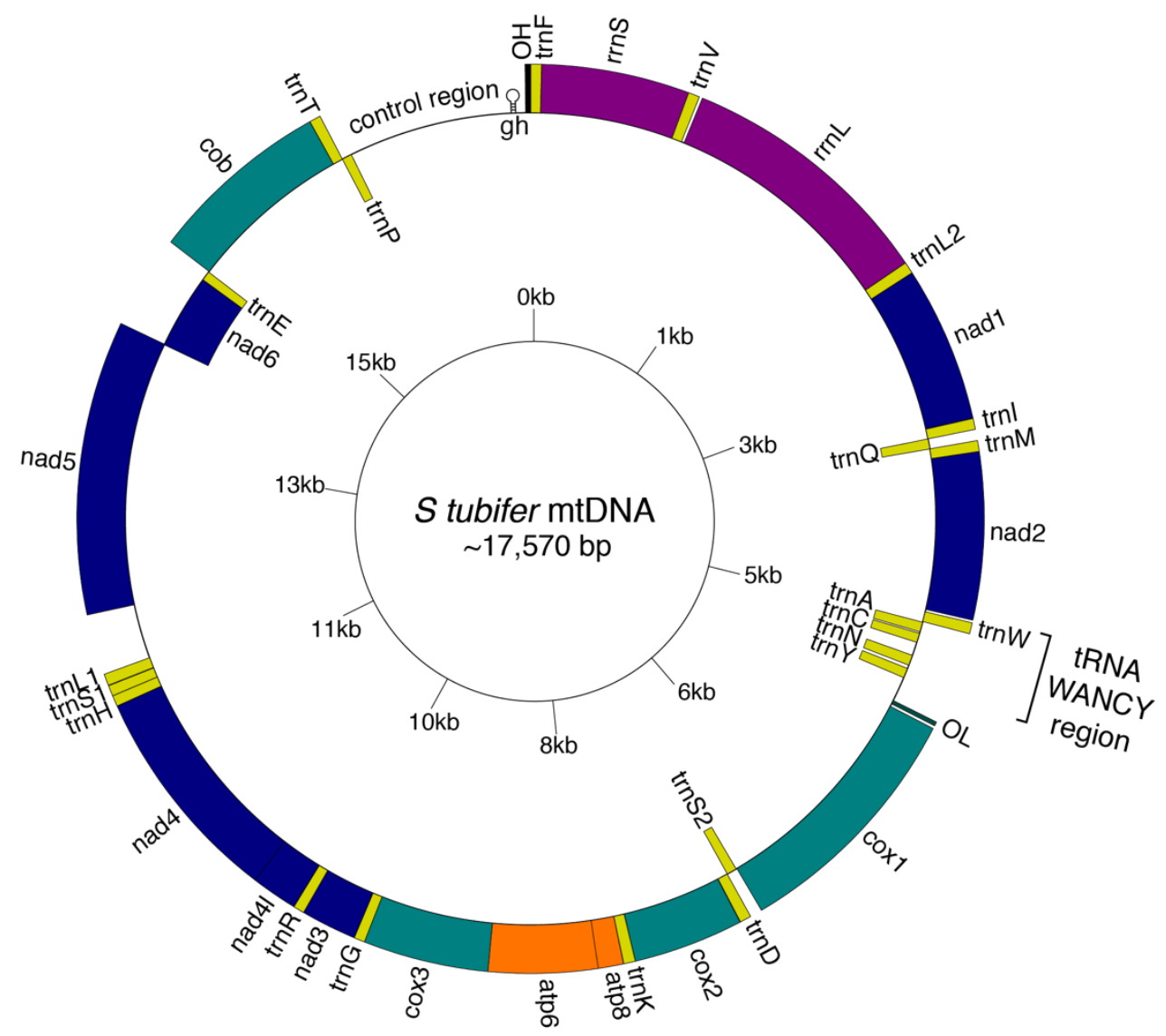

b.

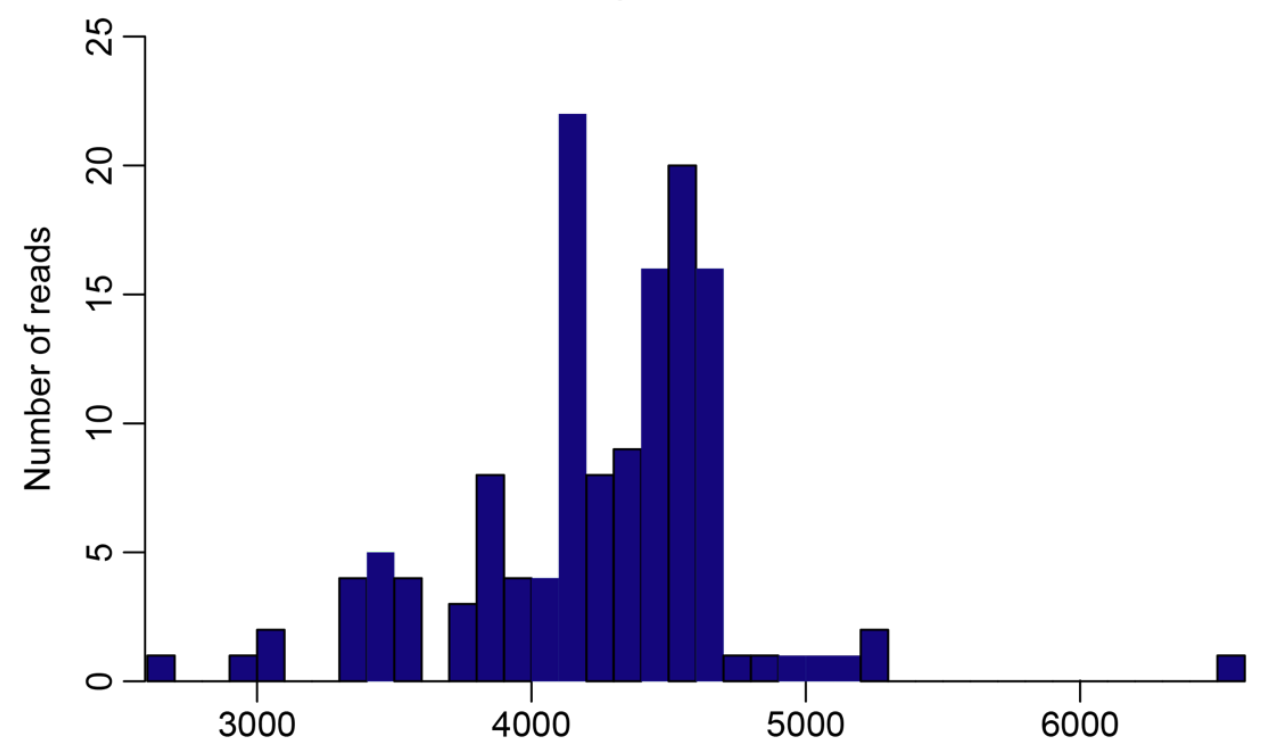

Figure 5. a) Gene map of the complete mitogenome of Siphamia tubifer. All genes are labelled in addition to the tRNA WANCY region, the control region, and the approximate location of the goose hairpin (gh) within the control region. b) Histogram depicting heteroplasmy in the length of the control region observed for the HiFi sequence reads spanning the entire region. 


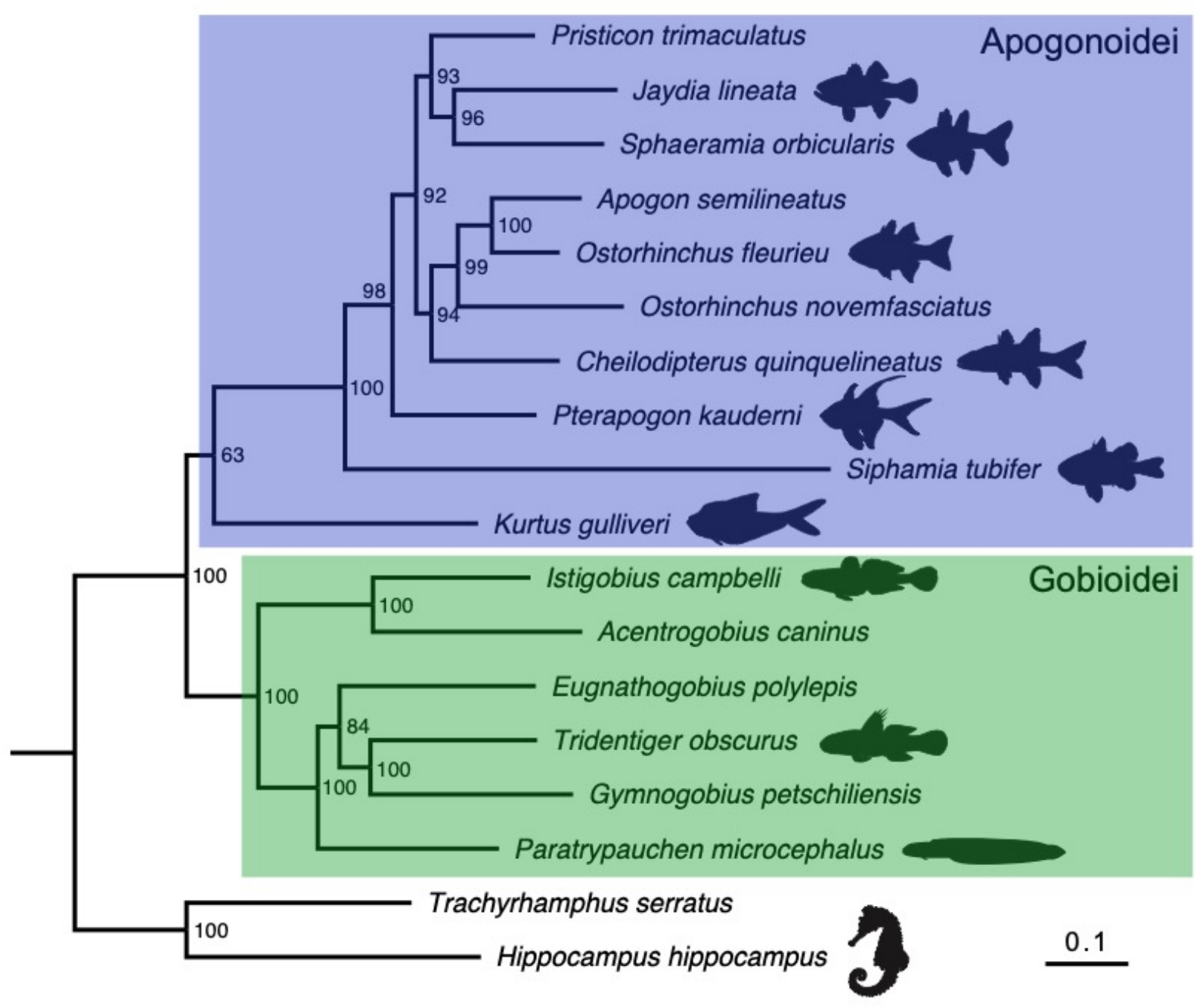

Figure 6. Maximum likelihood tree depicting the phylogenetic relationships of the cardinalfish species for which there is whole mitochondrial genome data available, including Siphamia tubifer from this study, in relation to another member of the Apogonoidei clade and several species of gobies. Two Syngnathiformes species are included as an outgroup. The relationships are based on whole mitochondrial DNA sequences excluding the control region using the $\mathrm{GTR}+\mathrm{F}+\mathrm{I}+\mathrm{G} 4$ model of substitution. Bootstrap support values (500 replicates) are listed at the nodes. The scale bar indicates nucleotide substitutions per site. The GenBank accession numbers for each species is listed in Table S3. 
506 Table 1. Assembly statistics of the draft genome of Siphamia tubifer.

\begin{tabular}{|ll|}
\hline Assembly size & $1,200,827,456 \mathrm{bp}$ \\
Total scaffolds & 118 \\
Total contigs & 2,057 \\
Scaffold N50 (L50) & $51,162,488 \mathrm{bp} \mathrm{(11)}$ \\
Contig N50 (L50) & $2,340,513 \mathrm{bp} \mathrm{(133)}$ \\
Scaffold N90 (L90) & $40,504,042 \mathrm{bp} \mathrm{(21)}$ \\
Contig N90 (L90) & 318,326 bp (689) \\
Total genes & 32,364 \\
Mean gene length & $12,504 \mathrm{bp}$ \\
\hline
\end{tabular}

507 
Table 2. Annotation statistics of the S. tubifer genome by chromosome. For each chromosome the total length in bp and the percent of those bp belonging to genes, introns, and exons are listed as well as the number of genes, introns, exons and tRNAs.

The mean length of genes, introns, and exons in each chromosome are also included.

\begin{tabular}{|c|c|c|c|c|c|c|}
\hline Chromosome & Length (bp) & $\begin{array}{c}\% \\
\text { Genes/Introns/Exons }\end{array}$ & Genes (mean bp) & $\begin{array}{l}\text { Introns (mean } \\
\text { bp) }\end{array}$ & Exons (mean bp) & tRNAs \\
\hline Chr1 & $57,520,444$ & $34.6 / 29.9 / 4.5$ & $1,622(12,267)$ & $12,401(1,386)$ & 14,023 (185) & 113 \\
\hline Chr2 & $35,111,521$ & $28.6 / 24.2 / 4.3$ & $906(11,080)$ & $6,733(1,263)$ & $7,639(195)$ & 271 \\
\hline Chr3 & $61,369,824$ & $30.6 / 26.9 / 3.6$ & $1,349(13,919)$ & $10,721(1,540)$ & $12,070(182)$ & 9 \\
\hline Chr4 & $57,984,278$ & $34.0 / 29.7 / 4.2$ & $1,503(13,119)$ & $12,691(1,355)$ & $14,194(172)$ & 329 \\
\hline Chr5 & $55,885,651$ & $31.8 / 27.5 / 4.2$ & $1,526(11,657)$ & $11,663(1,316)$ & 13,189 (177) & 769 \\
\hline Chr6 & $53,092,471$ & $37.4 / 32.2$ / 5.1 & $1,571(12,637)$ & $13,271(1,289)$ & $14,842(181)$ & 52 \\
\hline Chr7 & $50,849,673$ & $36.2 / 31.2 / 4.9$ & $1,523(12,094)$ & $12,644(1,255)$ & 14,167 (176) & 144 \\
\hline Chr8 & $49,165,404$ & $36.0 / 30.7 / 5.1$ & $1,620(10,931)$ & $12,232(1,233)$ & $13,852(182)$ & 77 \\
\hline Chr9 & $55,908,959$ & $38.4 / 32.9$ / 5.4 & $1,763(12,181)$ & $15,694(1,171)$ & 17,457 (171) & 192 \\
\hline Chr10 & $52,131,239$ & $23.9 / 20.3 / 3.5$ & $1,243(10,042)$ & $8,143(1,297)$ & $9,386(197)$ & 106 \\
\hline Chr11 & $49,759,318$ & 30.4 / 26.8 / 3.6 & $1,223(12,385)$ & $8,348(1,595)$ & $9,571(185)$ & 4 \\
\hline Chr12 & $77,671,243$ & $34.6 / 30.2$ / 4.2 & $2,000(13,424)$ & $16,721(1,402)$ & $18,721(172)$ & 70 \\
\hline Chr13 & $51,098,085$ & $32.9 / 28.4 / 4.3$ & $1,357(12,386)$ & $10,324(1,407)$ & $11,681(190)$ & 244 \\
\hline Chr14 & $51,888,355$ & $30.6 / 26.5 / 4.0$ & $1,302(12,212)$ & $9,713(1,413)$ & $11,015(188)$ & 284 \\
\hline Chr15 & $48,065,453$ & $36.5 / 32.0 / 4.3$ & $1,254(13,972)$ & $10,629(1,447)$ & $11,883(174)$ & 60 \\
\hline Chr16 & $62,834,756$ & $32.7 / 27.5 / 5.0$ & $1,912(10,735)$ & $14,667(1,179)$ & $16,579(190)$ & 110 \\
\hline Chr17 & $49,965,003$ & $39.5 / 34.6 / 4.7$ & $1,489(13,255)$ & $12,205(1,414)$ & $13,694(173)$ & 184 \\
\hline Chr18 & $33,955,268$ & $32.9 / 28.8 / 4.1$ & $862(12,965)$ & $6,429(1,522)$ & 7,291 (189) & 162 \\
\hline Chr19 & $41,814,943$ & $38.5 / 33.2 / 5.1$ & $1,318(12,199)$ & $10,149(1,366)$ & 11,467 (187) & 112 \\
\hline Chr20 & $40,504,042$ & $33.7 / 29.7$ / 3.8 & $967(14,124)$ & $7,995(1,506)$ & $8,962(171)$ & 7 \\
\hline Chr21 & $51,162,488$ & $38.4 / 32.8 / 5.5$ & $1,249(15,746)$ & $12,062(1,391)$ & 13,311 (209) & 22 \\
\hline
\end{tabular}




\begin{tabular}{|c|c|c|c|c|c|c|}
\hline Chr22 & $51,158,892$ & 34.9 / 29.9 / 4.7 & $1,389(12,853)$ & $11,809(1,296)$ & 13,198 (183) & 167 \\
\hline Chr24 & $41,094,201$ & $37.0 / 32.2 / 4.6$ & $1,130(13,442)$ & $9,247(1,431)$ & $10,377(184)$ & 19 \\
\hline Total & $1,200,827,456$ & 33.7 / $29.1 / 4.4$ & $32,365(12,504)$ & $257,898(1,356)$ & $290,263(183)$ & 3,507 \\
\hline
\end{tabular}

511

512 
513 Table 3. Gene ontology (GO) enrichment analysis for the 710 unique clusters identified in the

514 Siphamia tubifer genome from a three-way comparison of orthologous clusters with the non-

515 luminous cardinalfish Sphaeramia orbicularis and the mudskipper Periophthalmus

516 magnuspinnatus (order Gobiiformes).

\begin{tabular}{|c|c|c|c|}
\hline GO ID & Description & Count & p-value \\
\hline GO:0071625 & vocalization behavior & 6 & 7.13E-07 \\
\hline GO:0050808 & synapse organization & 8 & 8.31E-07 \\
\hline GO:0006310 & DNA recombination & 5 & $2.52 \mathrm{E}-06$ \\
\hline GO:0015074 & DNA integration & 4 & $6.32 \mathrm{E}-06$ \\
\hline GO:0030050 & vesicle transport along actin filament & 4 & $2.80 \mathrm{E}-05$ \\
\hline GO:0006313 & transposition, DNA-mediated & 4 & 4.91E-05 \\
\hline GO:0006953 & acute-phase response & 3 & 5.44E-04 \\
\hline GO:0075732 & viral penetration into host nucleus & 2 & 6.69E-04 \\
\hline GO:0032185 & septin cytoskeleton organization & 2 & 6.69E-04 \\
\hline GO:0001764 & neuron migration & 3 & $1.24 \mathrm{E}-03$ \\
\hline GO:0071805 & potassium ion transmembrane transport & 3 & 1.24E-03 \\
\hline GO:0034765 & regulation of ion transmembrane transport & 8 & 1.35E-03 \\
\hline GO:0003964 & RNA-directed DNA polymerase activity & 2 & 1.95E-03 \\
\hline GO:0043516 & $\begin{array}{l}\text { regulation of DNA damage response, signal transduction } \\
\text { by } p 53 \text { class mediator }\end{array}$ & 2 & 1.95E-03 \\
\hline GO:0044826 & viral genome integration into host DNA & 2 & 1.95E-03 \\
\hline GO:1900044 & regulation of protein K63-linked ubiquitination & 2 & 1.95E-03 \\
\hline GO:0032197 & transposition, RNA-mediated & 2 & 1.95E-03 \\
\hline GO:0047369 & succinate-hydroxymethylglutarate CoA-transferase activity & 2 & 1.95E-03 \\
\hline GO:0007522 & visceral muscle development & 2 & 1.95E-03 \\
\hline GO:0006261 & DNA-dependent DNA replication & 2 & 1.95E-03 \\
\hline GO:0090129 & positive regulation of synapse maturation & 2 & 1.95E-03 \\
\hline GO:0043551 & regulation of phosphatidylinositol 3-kinase activity & 2 & 1.95E-03 \\
\hline GO:0045162 & clustering of voltage-gated sodium channels & 2 & 1.95E-03 \\
\hline GO:0045214 & sarcomere organization & 4 & 2.03E-03 \\
\hline GO:0015031 & protein transport & 10 & $3.13 \mathrm{E}-03$ \\
\hline GO:0008154 & actin polymerization or depolymerization & 2 & 3.81E-03 \\
\hline
\end{tabular}




\section{References}

Altschul, S.F., Gish, W., Miller, W., Myers, E.W. \& Lipman, D.J. (1990) "Basic local alignment search tool." J. Mol. Biol. 215:403-410

Belcaid, M., Casaburi, G., McAnulty, S. J., Schmidbaur, H., Suria, A. M., Moriano-Gutierrez, S., Pankey, M.S., Oakley, T.H., Kremer, N., Koch, E.J., Collins, A.J... \& Nyholm, S. V. (2019). Symbiotic organs shaped by distinct modes of genome evolution in cephalopods. Proceedings of the National Academy of Sciences, 116(8), 3030-3035.

Belton, J. M., McCord, R. P., Gibcus, J. H., Naumova, N., Zhan, Y., \& Dekker, J. (2012). Hi-C: a comprehensive technique to capture the conformation of genomes. Methods, 58(3), 268-276.

Benson, G. (1999). Tandem repeats finder: a program to analyze DNA sequences. Nucleic acids research, 27(2), 573-580.

Bernt, M., Donath, A., Jühling, F., Externbrink, F., Florentz, C., Fritzsch, G., Pütz, J., Middendorf, M. \& Stadler, P.F., (2013). MITOS: improved de novo metazoan mitochondrial genome annotation. Molecular phylogenetics and evolution, 69(2), 313-319.

Brůna, T., Lomsadze, A., \& Borodovsky, M. (2020). GeneMark-EP+: eukaryotic gene prediction with self-training in the space of genes and proteins. NAR genomics and bioinformatics, 2(2), lqaa026.

Brůna, T., Hoff, K. J., Lomsadze, A., Stanke, M., \& Borodovsky, M. (2021). BRAKER2: protein database. NAR genomics and bioinformatics, 3(1), lqaa108.

Chakraborty, M., Baldwin-Brown, J. G., Long, A. D., \& Emerson, J. J. (2016). Contiguous and accurate de novo assembly of metazoan genomes with modest long read coverage. Nucleic acids research, 44(19), e147.

Chan, P. P., Lin, B. Y., Mak, A. J., \& Lowe, T. M. (2021). tRNAscan-SE 2.0: improved detection and functional classification of transfer RNA genes. Nucleic Acid Research. doi:10.1093/nar/gkab688 https://doi.org/10.1038/s41592-020-01056-5 
560

561

562

563

564

565

566

567

568

569

570

571

572

573

574

575

576

577

578

579

580

581

582

583

584

585

586

587

588

589

590

591

592

593

594

595

596

597

598

599

600

601

602

Aedes aegypti genome using Hi-C yields chromosome-length scaffolds. Science, 356(6333), 92-95.

Dudchenko, O., Shamim, M. S., Batra, S. S., Durand, N. C., Musial, N. T., Mostofa, R., Pham, M., St Hilaire, B.G., Yao, W., Stamenova, E. and Hoeger, M., ...\& Aiden, E. L. (2018). The Juicebox Assembly Tools module facilitates de novo assembly of mammalian genomes with chromosome-length scaffolds for under \$1000. BioRxiv, 254797.

Dunlap, P. V., \& Nakamura, M. (2011). Functional morphology of the luminescence system of Siphamia versicolor (Perciformes: Apogonidae), a bacterially luminous coral reef fish. Journal of morphology, 272(8), 897-909.

Dunlap, P. V., Gould, A. L., Wittenrich, M. L., \& Nakamura, M. (2012). Symbiosis initiation in the bacterially luminous sea urchin cardinalfish Siphamia versicolor. Journal of fish biology, 81(4), 1340-1356.

Durand, N. C., Robinson, J. T., Shamim, M. S., Machol, I., Mesirov, J. P., Lander, E. S., \& Aiden, E. L. (2016a). Juicebox provides a visualization system for $\mathrm{Hi}-\mathrm{C}$ contact maps with unlimited zoom. Cell systems, 3(1), 99-101.

Durand, N. C., Shamim, M. S., Machol, I., Rao, S. S., Huntley, M. H., Lander, E. S., \& Aiden, E. L. (2016b). Juicer provides a one-click system for analyzing loop-resolution Hi-C experiments. Cell systems, 3(1), 95-98.

Eibl-Eibesfeldt, I. (1961). Eine symbiose zwischen fischen (Siphamia versicolor) und seeigeln. Zeitschrift für Tierpsychologie, 18(1), 56-59.

Faber, J. E., \& Stepien, C. A. (1998). Tandemly repeated sequences in the mitochondrial DNA control region and phylogeography of the pike-perchesstizostedion. Molecular phylogenetics and evolution, 10(3), 310-322.

Farrer, R. A. (2017). Synima: a Synteny imaging tool for annotated genome assemblies. BMC bioinformatics, 18(1), 1-4.

Flynn, J. M., Hubley, R., Goubert, C., Rosen, J., Clark, A. G., Feschotte, C., \& Smit, A. F. (2020). RepeatModeler2 for automated genomic discovery of transposable element families. Proceedings of the National Academy of Sciences, 117(17), 9451-9457.

Formenti, G., Rhie, A., Balacco, J., Haase, B., Mountcastle, J., Fedrigo, O., Brown, S., Capodiferro, M.R., Al-Ajli, F.O., Ambrosini, R. and Houde, P.... \& Jarvis, E. D. (2021). Complete vertebrate mitogenomes reveal widespread repeats and gene duplications. Genome biology, 22(1), 1-22. 
603 Ghezelayagh, A., Harrington, R.C., Burress, E.D., Campbell, M.A., Buckner, J.C., Chakrabarty, 604 P., Glass, J.R., McCraney, W.T., Unmack, P.J., Thacker, C.E., Alfaro, M.E. . \& Near, T. J.

605 (2021). Prolonged morphological expansion of spiny-rayed fishes following the end-

606 Cretaceous.

607

608

609

Gon, O., \& Allen, G. R. (2012). Revision of the Indo-Pacific cardinalfish genus Siphamia

610

611 (Perciformes: Apogonidae). Zootaxa, 3294(1), 1-84.

612

Gould, A. L., \& Dunlap, P. V. (2017). Genomic analysis of a cardinalfish with larval homing potential reveals genetic admixture in the Okinawa Islands. Molecular ecology, 26(15), 38703882.

Gould, A. L., \& Dunlap, P. V. (2019). Shedding light on specificity: population genomic structure of a symbiosis between a coral reef fish and luminous bacterium. Frontiers in microbiology, 10, 2670.

Gould, A. L., Harii, S., \& Dunlap, P. V. (2014). Host preference, site fidelity, and homing behavior of the symbiotically luminous cardinalfish, Siphamia tubifer (Perciformes:

622

623 Apogonidae). Marine biology, 161(12), 2897-2907.

\section{4}

625

626

Gould, A. L., Harii, S., \& Dunlap, P. V. (2015). Cues from the reef: olfactory preferences of a symbiotically luminous cardinalfish. Coral Reefs, 34(2), 673-677.

Gould, A. L., Dougan, K. E., Koenigbauer, S. T., \& Dunlap, P. V. (2016). Life history of the symbiotically luminous cardinalfish Siphamia tubifer (Perciformes: Apogonidae). Journal of fish

\section{9}

630 biology, 89(2), 1359-1377.

\section{1}

Gould, A., Fritts-Penniman, A., \& Gaisiner, A. (2021). Museum genomics illuminate the high specificity of a bioluminescent symbiosis across a genus of reef fish. Frontiers in Ecology and Evolution, 9, 18.

Guan, D., McCarthy, S. A., Wood, J., Howe, K., Wang, Y., \& Durbin, R. (2020). Identifying and removing haplotypic duplication in primary genome assemblies. Bioinformatics, 36(9), 2896-

\section{7}

638

639 2898. mining segmental genome duplications and synteny. Bioinformatics, 20(18), 3643-3646.

Hoarau, G., Holla, S., Lescasse, R., Stam, W. T., \& Olsen, J. L. (2002). Heteroplasmy and 
645

646

647

648

649

650

651

652

653

654

655

656

657

658

659

660

661

662

663

664

665

666

667

668

669

670

671

672

673

674

675

676

677

678

679

680

681

682

683

684

685

686

687

Inoue JG, Miya M, Tsukamoto K, Nishida M. (2003). Evolution of the deep-sea gulper eel mitochondrial genomes: large-scale gene rearrangements originated within the eels. Mol Biol Evol. 20:1917-1924.

Iwai, T. (1958). A study of the luminous organ of the apogonid fish Siphamia versicolor (Smith and Radcliffe). Journal of the Washington Academy of Sciences, 48(8), 267-270.

Iwai, T. (1971). Structure of luminescent organ of apogonid fish, Siphamia versicolor. Japanese Journal of Ichthyology, 18(3), 125-127.

Jones, P., Binns, D., Chang, H. Y., Fraser, M., Li, W., McAnulla, C., McWilliam, H., Maslen, J., Mitchell, A., Nuka, G., Pesseat, S., ... \& Hunter, S. (2014). InterProScan 5: genome-scale protein function classification. Bioinformatics, 30(9), 1236-1240.

Jühling, F., Pütz, J., Bernt, M., Donath, A., Middendorf, M., Florentz, C., \& Stadler, P. F. (2012). Improved systematic tRNA gene annotation allows new insights into the evolution of mitochondrial tRNA structures and into the mechanisms of mitochondrial genome rearrangements. Nucleic acids research, 40(7), 2833-2845.

Kaeding, A. J., Ast, J. C., Pearce, M. M., Urbanczyk, H., Kimura, S., Endo, H., ... \& Dunlap, P. V. (2007). Phylogenetic diversity and cosymbiosis in the bioluminescent symbioses of "Photobacterium mandapamensis". Applied and Environmental Microbiology, 73(10), 31733182.

Karin, E. L., Mirdita, M., \& Söding, J. (2020). MetaEuk - sensitive, high-throughput gene discovery, and annotation for large-scale eukaryotic metagenomics. Microbiome, 8(1), 1-15.

Katoh, K., Misawa, K., Kuma, K. I., \& Miyata, T. (2002). MAFFT: a novel method for rapid multiple sequence alignment based on fast Fourier transform. Nucleic acids research, 30(14), 3059-3066.

Keller, O., Kollmar, M., Stanke, M., \& Waack, S. (2011). A novel hybrid gene prediction method employing protein multiple sequence alignments. Bioinformatics, 27(6), 757-763.

Kozlov, A. M., Darriba, D., Flouri, T., Morel, B., \& Stamatakis, A. (2019). RAxML-NG: a fast, scalable and user-friendly tool for maximum likelihood phylogenetic inference. Bioinformatics, 35(21), 4453-4455.

Kriventseva, E. V., Kuznetsov, D., Tegenfeldt, F., Manni, M., Dias, R., Simão, F. A., \& Zdobnov, E. M. (2019). OrthoDB v10: sampling the diversity of animal, plant, fungal, protist, bacterial and viral genomes for evolutionary and functional annotations of orthologs. Nucleic acids research, 47(D1), D807-D811. 
Krzywinski, M., Schein, J., Birol, I., Connors, J., Gascoyne, R., Horsman, D., Jones, S.J. and Marra, M.A. (2009). Circos: an information aesthetic for comparative genomics. Genome research, 19(9), 1639-1645.

Li, D., Liu, C. M., Luo, R., Sadakane, K., \& Lam, T. W. (2015). MEGAHIT: an ultra-fast singlenode solution for large and complex metagenomics assembly via succinct de Bruijn graph. Bioinformatics, 31(10), 1674-1676.

Li, H. (2018). Minimap2: pairwise alignment for nucleotide sequences. Bioinformatics, 34(18), 3094-3100.

Lieberman-Aiden, E., Van Berkum, N.L., Williams, L., Imakaev, M., Ragoczy, T., Telling, A., Amit, I., Lajoie, B.R., Sabo, P.J., Dorschner, M.O. and Sandstrom, R., ... \& Dekker, J. (2009). Comprehensive mapping of long-range interactions reveals folding principles of the human genome. science, 326(5950), 289-293.

Ludwig, A., May, B., Debus, L., \& Jenneckens, I. (2000). Heteroplasmy in the mtDNA control region of sturgeon (Acipenser, Huso and Scaphirhynchus). Genetics, 156(4), 1933-1947.

Marcais, G., \& Kingsford, C. (2012). Jellyfish: A fast k-mer counter. Tutorialis e Manuais, 1, 1-8.

Nguyen, L. T., Schmidt, H. A., Von Haeseler, A., \& Minh, B. Q. (2015). IQ-TREE: a fast and effective stochastic algorithm for estimating maximum-likelihood phylogenies. Molecular biology and evolution, 32(1), 268-274.

PacificBiosciences (2020). Ipa hifi genome assembler.

Poulsen JY, Byrkjedal I, Willassen E, Rees D, Takeshima H, Satoh TP, Shinohara G, Nishida M, Miya M. (2013). Mitogenomic sequences and evidence from unique gene rearrangements corroborate evolutionary relationships of Myctophiformes (Neoteleostei). BMC Evol Biol. 13:111

Poulsen, J. Y., Sado, T., \& Miya, M. (2019). Unique mitochondrial gene order in Xenodermichthys copei (Alepocephalidae: Otocephala)-a first observation of a large-scale rearranged 16S-WANCY region in vertebrates. Mitochondrial DNA Part B, 4(1), 511-514.

Quinn, T. W., \& Wilson, A. C. (1993). Sequence evolution in and around the mitochondrial control region in birds. Journal of molecular evolution, 37(4), 417-425.

Ranallo-Benavidez, T.R., Jaron, K.S. \& Schatz, M.C. GenomeScope 2.0 and Smudgeplot for reference-free profiling of polyploid genomes. Nature Communications 11, 1432 (2020). 
Rao, S.S., Huntley, M.H., Durand, N.C., Stamenova, E.K., Bochkov, I.D., Robinson, J.T., Sanborn, A.L., Machol, I., Omer, A.D., Lander, E.S. and Aiden, E.L. (2014). A 3D map of the human genome at kilobase resolution reveals principles of chromatin looping. Cell, 159(7), 1665-1680.

734

735

736

737

738

739

740

741

742

743

744

745

746

747

748

749

750

751

752

753

754

755

756

757

758

759

760

761

762

763

764

765

766

767

768

769

770

771

772

Samonte, I. E., Pagulayan, R. C., \& Mayer, W. E. (2000). Molecular phylogeny of Philippine freshwater sardines based on mitochondrial DNA analysis. Journal of Heredity, 91(3), 247-253

Simão, F. A., Waterhouse, R. M., loannidis, P., Kriventseva, E. V., \& Zdobnov, E. M. (2015). BUSCO: assessing genome assembly and annotation completeness with single-copy orthologs. Bioinformatics, 31(19), 3210-3212.

Smit, AFA, Hubley, R \& Green, P. RepeatMasker Open-4.0. 2013-2015

$<\underline{\text { http://www.repeatmasker.org }>}$

Tamura, R. (1982). Experimental observations on the association between the cardinalfish (Siphamia versicolor) and the sea urchin (Diadema setosum). Galaxea, 1, 1-10.

Thacker, C. E. (2014). Species and shape diversification are inversely correlated among gobies and cardinalfishes (Teleostei: Gobiiformes). Organisms Diversity \& Evolution, 14(4), 419-436.

Turanov, S. V., Lee, Y. H., \& Kartavtsev, Y. P. (2019). Structure, evolution and phylogenetic informativeness of eelpouts (Cottoidei: Zoarcales) mitochondrial control region sequences. Mitochondrial DNA Part A, 30(2), 264-272.

Urbanczyk, H., Ogura, Y., Hendry, T. A., Gould, A. L., Kiwaki, N., Atkinson, J. T., Hayashi, T. \& Dunlap, P. V. (2011). Genome sequence of Photobacterium mandapamensis strain svers. 1.1, the bioluminescent symbiont of the cardinal fish Siphamia versicolor.

Van Berkum, N.L., Lieberman-Aiden, E., Williams, L., Imakaev, M., Gnirke, A., Mirny, L.A., Dekker, J. and Lander, E.S. (2010). Hi-C: a method to study the three-dimensional architecture of genomes. JoVE (Journal of Visualized Experiments), (39), e1869.

Wada, M., Kamiya, A., Uchiyama, N., Yoshizawa, S., Kita-Tsukamoto, K., Ikejima, K., ... \& Kogure, K. (2006). Lux A gene of light organ symbionts of the bioluminescent fish Acropoma japonicum (Acropomatidae) and Siphamia versicolor (Apogonidae) forms a lineage closely related to that of Photobacterium leiognathi ssp. mandapamensis. FEMS microbiology letters, 260(2), 186-192.

Xu, L., Dong, Z., Fang, L., Luo, Y., Wei, Z., Guo, H., Zhang, G., Gu, Y.Q., Coleman-Derr, D., Xia, Q. and Wang, Y. (2019). OrthoVenn2: a web server for whole-genome comparison and annotation of orthologous clusters across multiple species. Nucleic acids research, 47(W1), W52-W58. 
774 Yoshiba, S., \& Haneda, Y. (1967). Bacteriological study on the symbiotic luminous bacteria

775 cultivated from the luminous organ of the apogonid fish, Siphamia versicolor and the Australian

776 pine cone fish, Cleidopus gloriamaris. Sci. Rep. Yokosuka City Mus, 13, 82-84.

777 